\title{
Crecimiento en montanera y características de la canal de cerdos ibéricos retinto del Andévalo
}

\author{
Forero, F.J. ${ }^{1}$; Venegas, M. ${ }^{1}$; Rey, A.I. ${ }^{2}$; López Bote, C.J. ${ }^{2}$ y Daza, A. ${ }^{3}$
}

'Diputación Provincial de Huelva. España.

2Departamento de Producción Animal. Facultad de Veterinaria. Universidad Complutense Ciudad Universitaria. Madrid. España.

${ }^{3}$ Departamento de Producción Agraria (Producción Animal). Escuela Técnica Superior de Ingeniería Agronómica, Alimentaria y de Biosistemas. Universidad Politécnica. Madrid. España.

\section{Palabras clave adicionales}

Efecto peso inicial.

Efecto peso canal.

\section{RESUMEN}

En un primer experimento se utilizaron tres grupos de cerdos lbéricos, machos castrados, de la línea Retinto del Andévalo que entraron en montanera con pesos diferentes: 88, 12 (peso bajo) 119,64 (peso intermedio) y 146,43 kg (peso alto) de peso medio respectivamente. Los cerdos de peso bajo e intermedio lograron un crecimiento diario significativamente superior que el de peso alto. De otra parte, en un segundo experimento, se recabaron los pesos al sacrificio de 100 cerdos de la línea genética precitada que fueron acabados en montanera en seis años consecutivos. Las canales se clasificaron según el peso al sacrificio (peso alto $>185 \mathrm{~kg}$, intermedio 165-185 kg y bajo $<165 \mathrm{~kg}$ ). El año tuvo influencia estadísticamente significativa sobre las características de la canal. El rendimiento canal fue significativamente más bajo en el grupo de cerdos sacrificado con peso bajo. El peso de los jamones, paletas y lomos aumentaron significativamente con el peso al sacrificio, pero el peso de los solomillos y la relación ponderal paletas/jamones no estuvieron afectados por el peso al sacrificio. El porcentaje en peso de los jamones, paletas, lomos y solomillos respecto al peso canal se redujo conforme aumentaba el peso canal. Los coeficientes alométricos de jamones paletas, lomos y solomillos respecto al peso canal fueron $0,563,0,479,0,422$ y 0,353 respectivamente. La relación ponderal paletas/jamones aumentaba hasta un peso de la canal de $134 \mathrm{~kg}$ y disminuía después con el aumento del peso canal. Se concluye que sería interesante sacrificar los cerdos en torno a $160 \mathrm{~kg}$ de peso vivo para aumentar la proporción de partes nobles en la canal y no penalizar la relación ponderal paletas/jamones.

\section{Growth on "Montanera" and carcass characteristics of"retinto del Andévalo" iberian pigs}

\section{SUMMARY}

\section{AdDITIONAL KEYWORDS}

Initial weight effect.

Carcass weight effect.

\section{INFORMATION}

\section{Cronología del artículo.}

Recibido/Received: 03.11.2016

Aceptado/Accepted: 22.02.2018

On-line: 15.04 .2018

Correspondencia a los autores/Contact e-mail:

argimiro.daza@upm.es

\section{INTRODUCCIÓN}

Según datos del MAGRAMA (2015) en mayo de 2015 España contaba con un censo de 298.858 reproductoras de la raza Ibérica de las cuales 22.448 se situaban en la provincia de Huelva Así mismo, en la fecha señalada, el censo nacional de cerdos para cebo, de más
In a first experiment three groups of Iberian pigs castrated males of "Retinto del Andévalo" line were used. At the beginning of free-range feeding "montanera" pigs weight was different: $88.12 \mathrm{~kg}$ (group of low weight), $119.64 \mathrm{~kg}$ (group of medium weight) and $146.43 \mathrm{~kg}$ (group of high weight). Low-weight and medium weight pig groups had an average daily gain higher than those high-weight pigs. On the other hand, in a second experiment, slaughter weights of "Retinto del Andévalo" line pigs, that were fattened under "montanera" conditions, were registered during consecutive six years $(n=100)$. The carcasses were classified according to slaughter weight (high weight $>185 \mathrm{~kg}$, medium weight from 165 to $185 \mathrm{~kg}$ and low weight $<165 \mathrm{~kg}$ ). The year had significant effect on carcass characteristics. The carcass yield was significantly lower in the pig group slaughtered with low weight. The weight of hams, forelegs and loins increased significantly with slaughter weight. Nevertheless, the slaughter weight had not significant influence on weight of sirloins, and forelegs weight/hams weight ratio. The percentage of hams, forelegs, loins and sirloins respect to carcass weight decreased as increased carcass weight. The alometric coefficients of hams, forelegs, loins and sirloins regarding carcass weight were $0.563,0.479$, 0.422 and 0,353 , respectively. The forelegs weight/hams weight ratio increased up to $134 \mathrm{~kg}$ of carcass weight and decreased afterwards for higher carcass weights. It is concluded that in pigs slaughter around $160 \mathrm{~kg}$ of live weight is interesting to increase the major joints proportion of the cascass and no penalize the foreleg weight/ham weight ratio. de $50 \mathrm{~kg}$, ascendía a 1.129 .882 animales de los cuales 44.410 estaban ubicados en Huelva. En la comarca del Andévalo de la citada provincia se ha desenvuelto tradicionalmente una fracción importante del censo del porcino Ibérico provincial (Junta de Andalucía 2001) bajo la denominación popular de Retinto del Andévalo explotado bajo fórmulas extensivas y semiextensivas 
de manejo de reproductores y animales durante la recría, y acabando a los cerdos con pienso o en montanera a pesos finales muy diversos. Algunos trabajos previos han aportado resultados productivos y características de la canal de cerdos pertenecientes a diversas variedades de la raza Ibérica que fueron acabados en montanera (Aparicio 1987; Benito 1996 ; Espárrago 1998; Barba et al. 2000 a y b ; Forero 1999; Espárrago et al. 2001; Forero 2008). Actualmente, todavía, a nuestro entender, no hay suficientes trabajos en la literatura, referida al cerdo Ibérico, sobre el crecimiento en montanera y calidad de la canal de cerdos de la línea Retinto del Andévalo. De otra parte, dentro del marco productivo del cerdo Ibérico, tampoco se ha estudiado suficientemente el efecto de un intervalo muy amplio de pesos de entrada de los cerdos en montanera sobre el crecimiento durante la misma y la influencia de un intervalo, también muy amplio, de pesos al sacrificio sobre la características de la canal. Dada la indudable importancia económica de los rendimientos de montanera y de la calidad de la canal del cerdo Ibérico (Espárrago 1998 ; Daza 2001) en este trabajo se estudia, en un primer experimento, el efecto del peso de entrada en montanera sobre los resultados productivos obtenidos en la misma y, en un segundo experimento independiente del anterior, la influencia del peso al sacrificio sobre las características de la canal de cerdos Ibéricos de la línea Retinto del Andévalo.

\section{MATERIAL Y MÉTODOS}

En un primer experimento, se utilizaron tres grupos de 17, 14 y 23 cerdos machos castrados de la línea Retinto del Andévalo pertenecientes a la finca "Huerto Ramírez" de la Diputación Provincial de Huelva. Los animales entraron en montanera con 88,12 (peso bajo) 119,64 (peso intermedio) y 146,43 kg (peso alto) (eem $=3,48 \mathrm{~kg}$ ) de peso medio respectivamente. Durante el periodo de premontanera los cerdos fueron alimentados en corrales con 2-2,5 $\mathrm{kg}$ de un pienso basado en harina de maíz-cebada. Los cerdos de peso bajo, intermedio y alto entraron en montanera con aproximadamente, 12, 13,5 y 14,5 meses de edad respectivamente. Cada grupo de cerdos correspondió a cada montanera de tres campañas consecutivas, de manera que coinciden el efecto año (campaña) y el efecto peso de entrada de los cerdos en montanera no pudiéndose, por lo tanto, separar estadísticamente ambos efectos.. La carga ganadera (entre 0,5 y 0,7 cerdos/ha) era ajustada anualmente para que los cerdos tuvieran disponi- bilidad de bellota ad líbitum. Los cerdos se pesaron en una báscula de alta precisión al comienzo y al final de la montanera y se controlaron las fechas de entrada y salida de la misma (Tabla I).

De otra parte, en un segundo experimento independiente del anterior, se recabaron los pesos al sacrificio de 100 cerdos de la línea genética precitada procedentes de la finca indicada que fueron acabados en montanera exclusiva en seis campañas consecutivas pero que no fueron controlados durante las mismas. Las canales se clasificaron según el peso al sacrificio (peso alto $>185 \mathrm{~kg}$, peso intermedio $165-185 \mathrm{~kg}$ y peso bajo $<165 \mathrm{~kg}$ ). Los cerdos, después de un ayuno próximo a 12 horas, se sacrificaron en el matadero de Villanueva de los Castillejos (Huelva) donde se obtuvieron los pesos individuales de la canal, jamones, paletas lomos y solomillos recortados izquierdos y derechos.

Los datos correspondientes a los resultados productivos obtenidos en montanera (primer experimento) se analizaron mediante un análisis de varianza que consideró como efecto principal a la clase de los cerdos según su peso de entrada en montanera. Mediante regresión simple se relacionaron las variables dependientes peso al sacrificio y ganancia media diaria con la variable independiente peso de entrada de los cerdos en montanera.

Los datos recabados en el matadero, correspondientes al segundo experimento, se analizaron mediante un análisis de varianza que consideró como efectos fijos el año o campaña y la clase de peso al sacrificio (alta, intermedia y baja) así como la interacción entre ambos factores. La simetría bilateral de jamones, paletas, lomos y solomillos de la canal se estudió mediante análisis de varianza y de correlación Así mismo, mediante regresión simple, se relacionaron el peso de las piezas de la canal con el peso al sacrificio y el porcentaje en peso de las mismas respecto al peso canal con el peso canal. Los coeficientes alométricos del peso canal respecto al peso al sacrificio y de las partes nobles de la canal respecto al peso canal fueron calculados mediante la ecuación: $y=$ a X b (Huxley, 1932), en donde " ${ }^{\prime}$ " fue el peso canal o de la parte noble correspondiente de la canal (jamones, paletas, lomos, solomillos y suma de las precitadas piezas) y $\mathrm{X}$ el peso al sacrificio (para el coeficiente alométrico de la canal) o el peso de la canal (para los coeficientes alométricos de las partes nobles de la canal) y b el coeficiente alométrico.

Tabla I. Efecto del peso de los cerdos a la entrada en montanera sobre el peso final y la ganancia media diaria (GMD) (Effect of pigs weight at the beginning of free-range feeding "montanera" on final weight and average daily gain).

\begin{tabular}{|c|c|c|c|c|c|}
\hline Peso inicial cerdos a la entrada en montanera & Alto & Intermedio & Bajo & eem & $\mathrm{P}<$ \\
\hline $\mathrm{n}$ & 23 & 14 & 17 & - & - \\
\hline Periodo de montanera & $30 / 11-12 / 02$ & $30 / 10-11 / 02$ & $30 / 09-05 / 02$ & & \\
\hline Días de montanera & 74 & 104 & 128 & - & - \\
\hline Peso inicial $(\mathrm{kg})$ & $146,43^{a}$ & $119,64^{b}$ & $88,12^{\mathrm{c}}$ & 3,48 & 0,0001 \\
\hline Peso final (kg) & 168,17 & 184,64 & 177,18 & 5,20 & 0,094 \\
\hline GMD (g) & $294^{b}$ & $625^{a}$ & $696^{a}$ & 29,90 & 0,0001 \\
\hline
\end{tabular}

$\mathrm{n}=\mathrm{n}^{\circ}$ de cerdos, eem = error estándar de la media. Medias con distintos superíndices difieren $\mathrm{P}<0,05$. 
Tabla II. Efecto del peso al sacrificio sobre las características de la canal (Effect of slaughter weight on carcass characteristics).

\begin{tabular}{|c|c|c|c|c|c|}
\hline Clase de PS & $\begin{array}{c}\text { Alto } \\
>185 \mathrm{~kg}\end{array}$ & $\begin{array}{l}\text { Intermedio } \\
165-185 \mathrm{~kg}\end{array}$ & $\begin{array}{c}\text { Bajo } \\
<165 \mathrm{~kg}\end{array}$ & eem & $\mathrm{P}$ clase de $\mathrm{PS}<$ \\
\hline $\begin{array}{l}\mathrm{n} \\
\mathrm{PS}(\mathrm{kg})\end{array}$ & $\begin{array}{c}37 \\
200,73^{a}\end{array}$ & $\begin{array}{c}34 \\
176,21^{b}\end{array}$ & $\begin{array}{c}29 \\
145,86^{c}\end{array}$ & 2,11 & 0,0001 \\
\hline $\mathrm{PC}(\mathrm{kg})$ & $171,5^{a}$ & $149,20^{b}$ & $119,91^{c}$ & 1,92 & 0,0001 \\
\hline $\mathrm{RC}(\%)$ & $85,27^{a}$ & $84,70^{a}$ & $82,30^{b}$ & 0,55 & 0,0007 \\
\hline PJ (kg) & $25,12^{a}$ & $22,71^{b}$ & $20,74^{c}$ & 0,34 & 0,0001 \\
\hline $\mathrm{PP}(\mathrm{kg})$ & $16,41^{\mathrm{a}}$ & $15,23^{b}$ & $13,87^{c}$ & 0,31 & 0,0001 \\
\hline $\mathrm{PL}(\mathrm{kg})$ & $3,95^{\mathrm{a}}$ & $3,69^{a b}$ & $3,55^{b}$ & 0,096 & 0,016 \\
\hline PJ/PP & 0,655 & 0,670 & 0,669 & 0,009 & 0,45 \\
\hline P So (kg) & 0,619 & 0,576 & 0,585 & 0,022 & 0,33 \\
\hline PSPN (kg) & $46,11^{\mathrm{a}}$ & $42,30^{b}$ & $38,79^{c}$ & 0,63 & 0,0001 \\
\hline \% Jamón ${ }^{1}$ & $14,70^{\mathrm{b}}$ & $15,27^{b}$ & $17,30^{\mathrm{a}}$ & 0,21 & 0,0001 \\
\hline$\%$ Paleta $^{1}$ & $9,63^{c}$ & $10,25^{b}$ & $11,58^{a}$ & 0,22 & 0,0001 \\
\hline$\%$ Lomo $^{1}$ & $2,30^{c}$ & $2,47^{b}$ & $2,96^{a}$ & 0,047 & 0,0001 \\
\hline$\%$ Solomillo $^{1}$ & $0,361^{c}$ & $0,450^{b}$ & $0,556^{a}$ & 0,013 & 0,0001 \\
\hline$\%$ Suma partes nobles ${ }^{1}$ & $27,00^{c}$ & $28,35^{b}$ & $32,37^{a}$ & 0,28 & 0,0001 \\
\hline
\end{tabular}

$\mathrm{n}=\mathrm{n}^{\circ}$ de canales, eem = error estándar de la media, $\mathrm{PS}=$ peso al sacrificio, $\mathrm{PC}=$ peso canal, $\mathrm{RC}=$ rendimiento canal. $\mathrm{PJ}=$ peso jamones, $\mathrm{PP}=$ peso paletas, $\mathrm{PL}=$ peso lomos, $\mathrm{PSo}=$ peso solomillos, $\mathrm{PSPN}=$ peso suma de partes nobles, respecto al peso canal. Medias con distintos superíndices difieren $P<0,05$.

La comprobación de la distribución normal de los datos obtenidos se realizó mediante el test de Shapiro y Wilk y cuando los datos de alguna variable no se ajustaron a tal distribución fueron transformados a arcsen $(\mathrm{x} / 100)^{0,5}$. Las medias obtenidas se compararon mediante el test de Scheffe. Todos los análisis se realizaron mediante la utilización del paquete estadístico SG Centurión.

\section{RESULTADOS}

\section{EXPERIMENTO 1: RESULTADOS PRODUCTIVOS DE MONTANERA}

Según puede observarse en la Tabla I los cerdos que entraron en montanera con pesos bajos lograron un crecimiento diario significativamente superior $(\mathrm{P}<0,0001)$ que los que entraron con pesos intermedios o altos y el peso final de sacrificio de los cerdos que iniciaron la montanera con pesos intermedios tendió a ser superior $(\mathrm{P}<0,10)$ que el de los que comenzaron la montanera con pesos bajos. La relación obtenida entre el peso al sacrificio (PS en $\mathrm{kg}$ ) y el peso inicial de entrada en montanera (PI en $\mathrm{kg}$ ) respondió a una función doble inversa de estructura:

$$
\begin{aligned}
\mathrm{PS}=1 /(0,0043+0,17 / \mathrm{PI}) \quad \mathrm{R}^{2}=0,20, \mathrm{RSD}=0,00083 \\
\mathrm{P}<0,0010
\end{aligned}
$$

De tal ecuación puede inferirse que el peso al sacrificio aumentó con el peso de entrada en montanera, pero el peso de entrada de los cerdos en montanera sólo explicó el $20 \%$ de la variabilidad del peso al sacrificio.

La relación entre la ganancia media diaria (GMD en $\mathrm{kg}$ ) y el peso de entrada en montanera (PI en $\mathrm{kg}$ ) se ajustó a una función lineal de estructura:
$\mathrm{GMD}=1,232-0,00584 \mathrm{PI}, \mathrm{R}^{2}=0,58, \quad \mathrm{RSD}=0,14$ , $\mathrm{P}<0,0001$

En tal ecuación el PI explicó el 58\% de la variabilidad de la GMD de los cerdos durante la montanera.

\section{EXPERIMENTO 2: CARACTERÍSTICAS DE LA CANAL}

Según los resultados reflejados en la Tabla II la clase de peso al sacrificio afectó significativamente a las características de la canal. El rendimiento a la canal fue significativamente más bajo en el grupo de cerdos sacrificado con peso bajo, y entre los grupos de cerdos sacrificados con peso intermedio y alto no se detectaron variaciones significativas de tal variable. Considerando el conjunto de todos los cerdos de este segundo experimento, el coeficiente alométrico del peso canal respecto al peso al sacrificio fue de 1,06. Como cabía esperar, el peso de los jamones, paletas, lomos y el de la suma de las partes nobles de la canal (jamones, paletas, lomos y solomillos) aumentaron significativamente con el peso al sacrificio, pero las variables peso de los solomillos y relación peso de las paletas/peso de los jamones no estuvieron afectados por la clase de peso al sacrificio. El porcentaje en peso de los jamones, respecto al peso canal, fue significativamente superior en el grupo de cerdos sacrificado a pesos bajos, no detectándose diferencias de estas variables entre los grupos de cerdos sacrificados con peso intermedio y alto. Sin embargo, los porcentajes de paletas, lomos y solomillos y el de la suma de partes nobles de la canal respecto al peso canal fueron significativamente diferente en los tres grupos de cerdos estudiados reduciéndose significativamente con el incremento del peso canal. El año de sacrificio no tuvo efecto estadísticamente significativo sobre el peso al sacrificio y el peso canal pero afectó significativamente $(\mathrm{P}<0,05)$ a las características de la 
Tabla III. Relaciones entre el rendimiento a la canal (RC) y el peso al sacrificio(PS y entre) el porcentaje de las partes nobles de la canal y de la relación peso de las paletas (PP) y peso de los jamones (PJ) con el peso canal. (Relationship between major joints percentage of the carcass and forelegs/hams ratio with carcass weight).

\begin{tabular}{lccc}
\hline Ecuación de regresión & $\mathrm{R}^{2}$ & $\mathrm{RSD}$ & $\mathrm{P}<$ \\
\hline RC $(\%)=77,35+0,0390 \mathrm{PS}$ & 0,17 & 2,40 & 0,0002 \\
$\% \mathrm{~J}=23,075-0,0498 \mathrm{PC}$ & 0,54 & 1,11 & 0,0001 \\
$\% \mathrm{P}=16,21-0,0390 \mathrm{PC}$ & 0,41 & 1,12 & 0,0001 \\
$\% \mathrm{~L}=10,129-1,52$ In PC & 0,43 & 0,30 & 0,0001 \\
$\%$ So $=0,688-0,00189 \mathrm{PC}$ & 0,25 & 0,078 & 0,0001 \\
$\%$ PSPN $=44,10-0,101 \mathrm{PC}$ & 0,57 & 2,15 & 0,0001 \\
PP/PJ $=0,446+0,00348 \mathrm{PC}-0,0000130 \mathrm{PC}^{2}$ & 0,06 & 0,050 & 0,05
\end{tabular}

$n=100$ observaciones, porcentajes respecto al peso canal, $P C=$ peso de la canal, $J=$ jamones, $P=$ paletas, $L=$ lomos, So $=$ solomillos, PSPN = peso suma de partes nobles. $R^{2}=$ coeficiente de determinación, $R S D=$ desviación residual estándar, $P=$ probabilidad

canal estudiadas, incluso cuando el peso canal era corregido introduciéndolo en el modelo estadístico como covariable.

En la Tabla III se exponen las relaciones entre el rendimiento a la canal y el peso al sacrificio y entre los porcentajes, respecto al peso canal, de jamones, paletas, lomos, solomillos y de la suma de estas cuatro partes de la canal con el peso canal. El rendimiento a la canal aumentó con el peso al sacrificio, pero el peso al sacrificio sólo explicó el 17\% de la variación del rendimiento a la canal. A medida que aumentaba el peso de la canal los porcentajes de las partes nobles de la misma disminuyeron significativamente, resultados que corroboran los obtenidos en el análisis de varianza. Los coeficientes alométricos de los jamones paletas, lomos solomillos y de la suma de las partes nobles de la canal respecto al peso canal fueron 0,563, 0,479, 0,422, 0,353 y 0,520 respectivamente. En la Tabla III también se muestra la relación entre el cociente peso de paletas / peso de jamones con el peso de la canal. Dicha relación se ajustó a una función cuadrática que fue significativa $(\mathrm{P}<0,05)$, aunque el peso de la canal sólo explicó el 6\% de la variabilidad de la relación señalada. Según la estructura de esta ecuación la relación paletas/jamones aumentaba hasta un peso de la canal próximo a $134 \mathrm{~kg}$, pero después, para pesos más elevados de la canal, tal relación se reducía. Hasta los $134 \mathrm{~kg}$ canal los coeficientes alométricos de paletas y jamones respecto al peso de la canal fueron significativamente diferentes $(0,956 \mathrm{y}$ 0,877 respectivamente, $\mathrm{P}<0,03$ ) pero después, para pesos superiores de la canal, el coeficiente alométrico de los jamones fue significativamente superior que el de las paletas $(0,549$ y 0,380 respectivamente, $\mathrm{P}<0,0001)$. Los coeficientes alométricos de las paletas respecto al jamón fueron de 1,777 y 0,750 en las canales de peso menor y mayor de $134 \mathrm{~kg}$ respectivamente.

Los resultados de la Tabla IV informan sobre los resultados concernientes a la simetría bilateral de las partes nobles de la canal. Como puede deducirse de los datos reflejados en dicha tabla los pesos de los jamones, paletas, lomos y solomillos izquierdos y derechos fueron estadísticamente similares. Sin embargo, cuando se estudió la simetría bilateral dentro de cada clase de peso al sacrificio se detectó que el peso de las paletas izquierdas de los cerdos de la clase de peso alto al sacrificio fue significativamente superior $(\mathrm{P}<0,05)$ que el de las paletas derechas $(8,4$ vs $8,0 \mathrm{~kg})$ y el peso de los solomillos derechos de los cerdos de las clases baja e intermedia de peso al sacrificio fue significativamente más elevado $(P<0,05)$ que el de los solomillos izquierdos (en los cerdos de clase baja 305 us $280 \mathrm{~g}$ y en los de la clase intermedia 295 vs $280 \mathrm{~g}$ respectivamente).

\section{DISCUSIÓN}

\section{EXPERIMENTO 1: RESULTADOS PRODUCTIVOS DE MONTANERA}

Los cerdos que entraron en montanera con pesos altos tuvieron unas necesidades de mantenimiento y de crecimiento más elevadas que los que entraron con pesos bajos (FEDNA 2013), por lo que su crecimiento medio diario durante la montanera se redujo significativamente. La ganancia en montanera observada en este experimento concuerda con las obtenidas por Barba et al. (2000 a) y por Forero $(1999,2008)$ en varias estirpes de cerdo Ibérico y con la encontrada por Rodríguez-Estévez et al. (2007) en la variedad Silvela. Benito (1996) informó sobre ganancias medias diarias entre

Tabla IV. Análisis de la simetría bilateral de las piezas nobles de la canal (Analysis of the bilateral symmetry of carcass major joints).

\begin{tabular}{lccccc}
\hline & Izquierdo/a & Derecho/a & eem & $\mathrm{P}<$ & $\mathrm{r}$ \\
\hline Jamones $(\mathrm{kg})$ & 11,49 & 11,54 & 0,133 & 0,74 & 0,96 \\
Paletas $(\mathrm{kg})$ & 7,65 & 7,62 & 0,113 & 0,87 & 0,70 \\
Lomos $(\mathrm{kg})$ & 1,86 & 1,88 & 0,030 & 0,70 & 0,88 \\
Solomillos $(\mathrm{kg})$ & 0,292 & 0,303 & 0,0066 & 0,23 & 0,83 \\
\hline
\end{tabular}

$\mathrm{n}=100$ observaciones, eem = error estándar de la media $r=$ coeficiente de correlación entre los pesos de las piezas simétricas 
850 y 950 g para cerdos de varias estirpes sacrificados con pesos próximos a los $160 \mathrm{~kg}$, valores superiores a los observados en este experimento. Daza et al. (2007a) encontraron que cerdos de la misma edad, de la estirpe Torbiscal, reducían el crecimiento diario en montanera a medida que aumentaba el peso y edad de acceso a la misma. Se ha demostrado que un aumento de la edad de entrada de los cerdos en montanera tiene un efecto positivo sobre la ganancia diaria, pero si el peso de los animales al comienzo de la montanera es similar (Daza et al. 2006 a ; Daza et al. 2007 b). Según los autores precitados, para pesos de entrada similares en montanera un aumento de la edad de los cerdos conduce a un incremento de la capacidad de ingestión de bellota y hierba y a un crecimiento compensatorio derivado de la restricción de la alimentación durante el periodo de premontanera, lo que se traduce en un aumento de la ganancia media diaria. En nuestro experimento no se observó tal resultado porque los cerdos de peso intermedio y alto que entraron en montanera con más edad también entraron con un peso vivo significativamente superior que los de peso bajo, como consecuencia de no haber sido restringidos severamente durante el periodo de premontanera.

\section{EXPERIMENTO 2: CARACTERÍSTICAS DE LA CANAL}

Los rendimientos a la canal obtenidos en nuestro experimento están de acuerdo con los obtenidos por Barba et al. (2000b) en seis estirpes de cerdo Ibérico. A medida que aumenta el peso vivo, la canal crece más rápidamente que el aparato digestivo y los cerdos sintetizan más grasa (Whittemore 1993), por lo que el rendimiento a la canal aumenta con el peso al sacrificio. En nuestro experimento una diferencia de peso al sacrificio próxima a $25 \mathrm{~kg}$ no se tradujo en una diferencia estadísticamente significativa de rendimiento a la canal. En cerdos Ibéricos sacrificados entre 115 y $170 \mathrm{~kg}$, sorprendentemente, Aparicio (1987) no detectó variaciones significativas del rendimiento a la canal ni tampoco Dobao et al. (1985) entre 100 y 118 kg, 103 y $137 \mathrm{~kg}$ y 117 y $148 \mathrm{~kg}$ en las estirpes Guadyerbas, Gamito y Torbiscal respectivamente. Diéguez (1992) encontró rendimientos a la canal entre el 83,1 y 84,4 \% en cerdos acabados en montanera y el coeficiente de correlación, no significativo, entre el peso al sacrificio y el peso de la canal fue tan sólo de $r=0,25$ valor inferior al detectado en nuestro experimento $(r=0,41)$. Como en nuestro experimento, De Pedro (1987) encontró variaciones significativas del rendimiento canal entre grupos de cerdos con más de $25 \mathrm{~kg}$ de diferencia de peso medio al sacrificio. El coeficiente alométrico del peso canal respecto al peso al sacrificio fue superior a la unidad, lo que concuerda con los valores obtenidos por De Pedro (1987). Por ello, parece lógico que el rendimiento canal aumente con el peso al sacrificio. De la relación obtenida entre rendimiento canal y peso al sacrificio se infiere que por cada $10 \mathrm{~kg}$ de aumento de peso al sacrificio el rendimiento canal aumenta un $0,39 \%$ variación similar a la obtenida por Latorre et al (2008) en cerdos blancos pesados destinados a la producción de productos elaborados, aunque otros autores en cerdos blancos pesados han observado variaciones superiores (Latorre et al. 2004).
Los valores de los pesos absolutos y de las proporciones de jamones y paletas respecto al peso canal concuerdan con los observados por Barba et al. (2000b). Óvilo et al. (2006) encontraron en 913 cerdos Ibéricos de varias ganaderías y estirpes, que fueron sacrificados con un peso medio de $162,37 \mathrm{~kg}$, pesos y proporciones similares de jamones y paletas que los obtenidos en este experimento en el grupo de cerdos de bajo peso al sacrificio, aunque en nuestro caso encontramos mayor cantidad de lomo. Para un peso al sacrifico de $167 \mathrm{~kg}$ León Ramírez et al. (2012b) en cerdos machos de la estirpe Silvela acabados en montanera obtuvieron 21,6 y 14,4 kg de jamón y paleta y rendimientos del 15,97 y $10,64 \%$ respecto al peso canal respectivamente, valores acordes con los observados en este experimento. Para pesos de la canal, de cerdos de montanera, comprendidos entre 112 y 197 kg, León Ramírez et al. (2012 a) encontraron un peso de los solomillos entre 0,40 y 0,89 $\mathrm{kg}$ y porcentajes respecto al peso canal entre el 0,28 y $0,65 \%$.

La campaña de montanera afectó a las características de la canal a pesar de haber corregido el peso canal. El efecto año o campaña incluye un conjunto muy amplio de factores y de sus interacciones que pueden influir en las variables de crecimiento $y$, por ende, en las características de la canal que no han sido controlados en el presente experimento. Así, son ejemplos ilustrativos que pueden haber influido en la variabilidad observada de las características de la canal: aspectos genéticos individuales inherentes a la conformación de los parentales dentro de una misma línea (Villafuerte et al. 2012), factores nutritivos relacionados con la alimentación durante la recría (Rodríguez Estévez et al. 2007), duración de la montanera y la disponibilidad de bellota y hierba, tamaño de la bellota y tenor de fibra de la ración (Durán y Lizaso 1997; Daza et al. 2008), factores de manejo durante la montanera como la edad y peso de entrada en la misma (Daza et al. 2006a) y durante el presacrificio como el transporte y la duración del ayuno) (Daza et al., 2016 datos no publicados), manipulación de las canales y piezas en el matadero (León Ramírez et al . 2012 b), factores sanitarios y ligados al ejercicio físico (Pugliese et al. 2003 y 2004) y condiciones ambientales durante la montanera (López Bote et al. 2001) y de la cámara de refrigeración (Lebret et al. 2001).

En el presente experimento, el porcentaje de partes nobles de la canal respecto al peso canal se redujo conforme aumentaba el peso canal lo que concuerda con los resultados de Espárrago (1998), Espárrago et al. (2001) y Daza et al. (2006b). En el presente experimento por cada $10 \mathrm{~kg}$ de aumento del peso canal los porcentajes de jamones y paletas se redujeron casi un 0,5 y un $0,39 \%$ respectivamente. Como quiera que los coeficientes alométricos de las partes nobles de la canal son menores de la unidad, es decir que aumentan de peso menos que el conjunto de la canal, conforme se incrementa el peso de la canal, parece lógico que el rendimiento de partes nobles se reduzca conforme aumentan el peso canal.

La relación entre el peso de las paletas y de los jamones es una variable de especial relevancia en el escenario comercial de la industria del cerdo Ibérico que 
no ha sido tratada suficientemente por la investigación porcina. Paletas demasiado pequeñas tiene dificultades de comercialización, por lo que la industria está muy interesada en un aumento de la relación ponderal paletas/jamones (Espárrago et al. 2001). Aunque en el presente experimento el peso al sacrificio no afectó a la citada relación, sin embargo, hemos detectado que estuvo relacionada significativamente con el peso de la canal mediante una función cuadrática en la que tal relación aumentaba hasta un determinado valor del peso canal $(134 \mathrm{~kg})$ para descender posteriormente para pesos de la canal superiores. Tal relación se explica porque, en nuestro experimento, hasta los $134 \mathrm{~kg}$ de peso canal el coeficiente alométrico de las paletas fue superior al de los jamones y para pesos superiores de la canal el coeficiente alométrico de los jamones fue muy superior al de las paletas. Este resultado fue corroborado con el cálculo del coeficiente alométrico de las paletas respecto a los jamones, Como ha sido indicado, este coeficiente fue superior a la unidad $(1,177)$ hasta los $134 \mathrm{~kg}$ de peso canal, lo que significa que, hasta este peso canal, las paletas crecen más rápidamente que los jamones, pero después, a peso canal superiores, ocurre lo contrario: los jamones crecen más rápidamente que las paletas (coeficiente alométrico 0,751). Este resultado, a nuestro juicio, es interesante en lo que concierne a la preocupación de la industria por el peso comercial de las paletas.

La ausencia de diferencias significativas entre los pesos de jamones y paletas izquierdos y derechos también fue evidenciada en cerdos Ibéricos por Barba et al. (2000b) y por Tejerina y García (2012), aunque no hemos encontrado información que haya estudiado la simetría bilateral de lomos y solomillos. Las diferencias encontradas, en este experimento entre el peso de las paletas derechas e izquierdas, en el grupo de cerdos del peso alto al sacrificio, y entre los solomillos derechos e izquierdos en los grupos de cerdos de peso al sacrificio intermedio y bajo pudieron estar ligadas a la operación de recortado de las mismas.

\section{CONCLUSIONES}

El aumento excesivo de peso de los cerdos a la entrada de montanera conduce a una reducción de la ganancia diaria durante dicho segmento productivo. Aumentar el peso al sacrificio por encima de los 160-165 kg de peso vivo (lo que equivale aun peso canal próximo a $134 \mathrm{~kg}$ ) se traduce en una reducción significativa de los porcentajes ponderales de las partes nobles de la canal respecto al peso canal. Sacrificar cerdos Ibéricos de la línea Retinto del Andévalo por encima del intervalo de pesos precitado no conduce a un aumento significativo de la relación peso de las paletas/peso de los jamones, ya que la velocidad de crecimiento de las paletas es inferior, cuando se coteja con la de los jamones, para pesos superiores del intervalo de peso señalado. No siempre se observa simetría bilateral ponderal de las partes nobles de la canal en el cerdo Ibérico. El peso canal la manipulación de la misma puede ser un factor de influencia, por lo que en los estudios experimentales, esta consideración debe ser tenida en cuenta.

\section{AGRADECIMIENTOS}

A la Diputación de Huelva sin cuyo apoyo no habría sido posible la realización de este trabajo.

\section{BIBLIOGRAFÍA}

Aparicio, J 1987, El cerdo Ibérico, Ed Sánchez Romero Carvajal, Huelva, España.

Barba, C, Delgado, J, Sereno, R, Diéguez, E \& Cañuelo, P 2000 a, 'Caracterización productiva de las variedades de cerdo Ibérico. I: Estudio preliminar de los pesos y crecimientos en premontanera y montanera', Archivos de Zootecnia, vol. 49, no 185-186, pp. 179-187.

Barba, C, Delgado, J, Diéguez, E \& Cañuelo, P 2000 b, 'Caracterización productiva de las variedades de cerdo lbérico basada en el comportamiento en matadero'. Archivos de Zootecnia, vol. 50, pp. 125-132.

Benito, J 1996, 'Las bases de la explotación extensiva del cerdo Ibérico', en C Buxadé (ed.), Zootecnia. Bases de la Producción Animal, Mundi Prensa, Madrid, España., pp. 316-331.

Daza, A 2001 'Bases del análisis económico y financiero', en C Buxadé \& A Daza (ed.), Porcino Ibérico: aspectos claves, Mundi Prensa, Madrid, España., pp. 689-720.

Daza, A, López Bote, C, Rey, A \& Olivares, A 2006 a, 'Effect of age at the beginning of the free-range period on growth and carcass and fat quality in Iberian pigs', Archives of Animal Nutrition, vol. 60, pp. 1-8.

Daza, A, Mateos, A, López Carrasco, C, Rey, A, Ovejero, I \& López Bote, C 2006 b, 'Effect of feeding system on the growth and carcass characteristics of Iberian pigs, and the use of ultrasound to estimate yields of joints', Meat Science, vol. 72, pp. 1-8.

Daza, A, Mateos, A, Rey, A , Ovejero, I \& López Bote, C 2007 a, 'Effect of duration of feeding under free-range conditions on production results and carcass and fat quality in Iberian pigs', Meat Science, vol. 76, pp. 411-416.

Daza, A, López Bote, C, Olivares, A, Menoyo, D \& Ruiz, J 2007 b, 'Age at the beginning of the fattening periodo $f$ lberia pigs under free-range conditions affects growth, carcass characteristics and the fatty acid profile of lipids', Animal Feed Science and Technology, vol. 139, pp. 81- 91.

Daza, A, Rey, A, López Carrasco, C \& López Bote, C 2008, 'Influence of acorn size on growth performance, carcass quality and fatty acid composition of subcutaneous and intramuscular fat from lberian pigs fattened in confinement', Spanish Journal of Agricultural Research, vol. 6, pp. 230-235.

De Pedro, E1987, 'Estudio de los factores sexo y peso al sacrificio sobre las características de la canal del cerdo Ibérico', Tesis Doctoral, Universidad de Córdoba, Córdoba, España.

Diéguez, E 1992, 'Cría del cerdo Ibérico', Mundo Ganadero, no. 9, pp. 25-32.

Dobao, M, Poza M, Rodrigáñez, J \& Silió, L 1985, 'Diferencias en la composición de la canal de tres estirpes de cerdo lbérico', Anales del INIA.Serie Ganadera, vol. 22, pp. 99-112.

Durán, R \& Lizaso, J 1997, 'Alimentación del cerdo Ibérico', Anaporc, no. 170 , pp. 82-106.

Espárrago, F 1998, 'Estudio comparativo de calidad, rendimiento y costes entre cerdos Ibéricos puros y cruzados engordados en régimen de montanera', Solo Cerdo Ibérico, no.1, pp.108-118.

Espárrago, F, Cabeza de Vaca, F, Molina, M \& Guijarro, J 2001. 'La industria del cerdo Ibérico' en C Buxadé \& A Daza (ed.), Porcino lbérico: aspectos claves, Mundi Prensa, Madrid, España., pp. 535-588.

FEDNA, 2013, Necesidades nutricionales para ganado porcino: Normas Fedna, $3^{a}$ ed, Fundación Española para el Desarrollo de la Nutrición Animal, Universidad Politécnica de Madrid, Madrid, España.

Forero, F 1999, Estudio comparativo de cinco estirpes de cerdo Ibérico, Diputación Provincial de Huelva, Huelva, España.

Forero, F 2008, 'La raza Ibérica. Principales variedades', en F Forero (ed.), El cerdo Ibérico. Una revisión transversal, Junta de Andalucía- Consejería de Agricultura y Pesca, Sevilla, España., pp. 65-82.

Huxley, J 1932, Problems of relative growth, Ed Methuen, London, UK. 
Junta de Andalucía, 2001, Caracterización del territorio de la OCA "Andévalo Oriental", Junta de Andalucía. Consejería de Agricultura y Pesca, Sevilla, España.

Latorre, M, Lázaro, R, Valencia, D, Medel, P \& Mateos, G 2004, 'Effect of gender and slaughter weight on the growth performance, carcass traits and meat quality characteristics of heavy pigs', Journal of Animal Science, vol. 82, pp. 526-533.

Latorre, M, García-Berenguer, E \& Ariño, L 2008, 'The effect of sex and slaughter weight on growth performance and carcass traits of pigs entended for dry-cured ham from Teruel (Spain)' Journal of Animal Science, vol. 86, pp. 1933-1942.

Lebrel, B, Yuin, H, Noblet, J \& Bonneau, M 2001, 'The effects of two methods of increasing age at slaughter weight on carcass and muscle traits and meat sensory quality in pigs', Animal Science, vol. 72, pp. 87-94.

León Ramírez, R, Rodríguez Estévez, V, Galán, H, López Rivero, J \& Rodríguez Sánchez, M 2012 a, 'Carcass performance of fresh meat pieces: sirloin, "presa" and "secreto" in Iberian pig finished at montanera', Options méditerranéennes, Series A, pp. 493-495.

León Ramírez, R, Rodríguez Estévez, V, Díaz Gaona, C \& Rodríguez Sánchez, M 2012 b, 'Sex influence in carcass (hams and shoulders) performance of lberian pigs fattened at montanera', Options méditerranéennes, Series A, pp. 507-510.

López Bote, C, Rey, A \& Isabel, B 2001, 'Alimentación del cerdo Ibérico en la Dehesa' en C Buxadé \& A Daza (ed.), Porcino lbérico: aspectos claves, Mundi Prensa, Madrid, España., pp. 215-246.
MAGRAMA, 2015, Anuario de Estadística Agroalimentaria, MAGRAMA, Madrid, España.

Pugliese, C, Madonia, G, Chiofalo, V, Margiotta, S, Acciaioli, A \& Gandini. G 2003, 'Comparison of the performance of Nero Siciliano pigs reared indoors and outdoors. 1 Growth and carcass composition', Meat Science, vol. 65, pp. 825-831.

Pugliese, C, Calagna, G, Chiofalo, V, Moretti, M, Margiotta, S, Franci O \& Gandini, G 2004, Comparison of the performance of Nero Siciliano pigs reared indoors and outdoors. 2 Joints composition, meat and fat traits, Meat Science, vol. 68, pp. 523-528.

Rodríguez-Estévez, V, Félix, E, Perea, J, Gómez, G, Mata, C \& García, A 2007, 'Ganancia media diaria en montanera de cerdos Ibéricos (Silvela) en función del sistema de recría', Archivos de Zootecnia, vol. 56, pp. 541-544.

Tejerina, D \& García Torres, S 2012, 'Effect of production system and sex on different carcass traits of Iberian pigs', Options méditerranéennes, Series A, pp. 401-404.

Villafuerte, S, Utrilla, M, García, A \& Soriano, A 2012, 'Differences in productive parameters and carcass traits of three sires from Duroc x Iberian cross selected according to their conformation', Options méditerranéennes, Series A, pp. 85-88.

Whittemore, C 1993. The science and practice of pig production, Logman Scientific and Technical Group UK Limited, London, UK. 